\title{
THICK GRATING ANALYSIS OF INTERFEROMETRICALLY PRODUCED WAVEGUIDES
}

\author{
E. LEITH, G. KUNG, R. ALFERNESS and B. HANSCHE \\ Dept. of Electrical and Comp. Engineering, University of Michigan, Ann Arbor, Michigan, USA
}

Received 18 February 1975 , revised manuscript received 10 April 1975

The thin-grating decomposition method of thick grating analysis is used to analyze guiding effects in thick phase gratings having sinusoidal refractive index modulation.

Interferometrically-produced phase gratings can be used as wave-guiding structures, as demonstrated by Rosenberg and Chandross [1]. Rosenberg gives an analysis of this phenomenon by ray tracing methods [2]. We note that the structure that produces Bragg diffraction is the same one that produces guiding, except that for guiding to be manifested, both the thickness and index modulation must be relatively large. We also note that the basic Bragg diffraction equation and the basic equation describing guiding in a slab waveguide are similar. We have therefore sought a single analysis, preferably a grating analysis based on physical optics, that describes both phenomena, and that will give insight into the transition region, when, with increasing refractive index modulation, the Bragg diffraction process produces confinement.

In the diffraction process, a plane wave impinges on the thick grating at the Bragg angle (fig. 1). When the wave enters the structure, a diffracted wave is formed; as the two waves propagate through the structure; there is a continuing exchange of energy between them. Continuing with this heuristic approach, we expect that when most or all of the energy in a plane wave is reflected from the first Bragg plane encountered, energy initially contained between two Bragg planes is confined between these planes until the wave emerges from the structure. Unfortunately, the usual analyses for diffraction from thick gratings assume uniform plane waves. The analyses predict the intensity of the emerging waves, but give no insight into the paths that the light traveled. In addition, the closed-form solutions are restrictive;

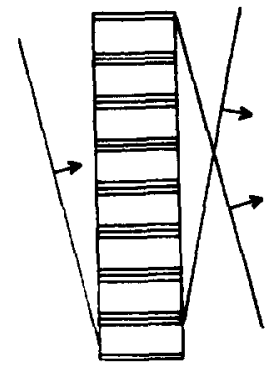

Fig. 1. Sinusoidal thick grating, showing incident and diffracted waves.

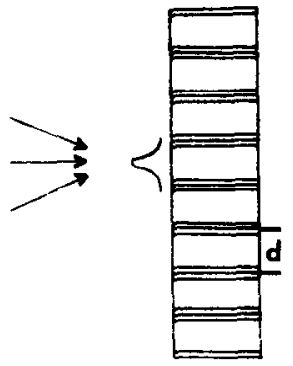

Fig. 2. Spatial light pulse incident on grating surface.

Kogelnik's coupled wave analysis [3], for example, allows for only two plane waves, and these must each be at or near the Bragg angles. We require an analysis that enables us to propagate a small spot of light (a spatial pulse) through the structure.

Alferness [4] has described an extremely general method, which allows the plane waves to be incident at any angle. It is, however, a plane-wave analysis, but it can be modified for our requirement. This method is called the thin grating decomposition meth. od, and is conceptually quite simple, yet in all tests 
that have been made, both by way of experiment and by comparison with special cases that can be analysed by other methods, the Alferness method has proved accurate. The method involves decomposing a thick grating into a stack of thin gratings, each of which is sufficiently thin that thin grating theory holds. Each thin grating diffracts the incident plane waves into various orders, depending on the grating structure. These diffracted waves are the input for the next thin layer. Typically, the decomposition involves about 30 to 100 thin gratings; thus, the analysis requires a computer.

In our procedure (fig. 2), a spatial pulse impinges on the source-side surface of the grating. The pulse is Fourier-decomposed into a spectrum of plane waves at various angles. The Alferness theory permits us to propagate each one through the structure, and to combine all of the emerging waves, both direct and diffracted, to find the resultant intensity distribution.

Since for calculational purposes we are restricted to a finite number of plane waves, we should choose an amplitude profile for the pulse which is not only realistic, but which can also be described without an excessive number of components. A suitable waveform is

$f(x)=\left(\frac{1}{2}+\frac{1}{2} \cos 2 \pi x / d\right)$ rect $x / d$,

where $d$ is the grating period, and rect $x / d=1$ for $|x| \leqslant \frac{1}{2} d$, and is zero otherwise. This waveform has the advantage that not only is it confined between the limits $|x|=\frac{1}{2} d$, but also that its spatial-frequency spectrum

$$
\begin{aligned}
& F\left(f_{x}\right)=\frac{1}{2} d \operatorname{sinc} d f_{x}+\frac{1}{4} d \operatorname{sinc} d\left(f_{x}+1 / d\right) \\
& \quad+\frac{1}{4} d \operatorname{sinc} d\left(f_{x}-1 / d\right)
\end{aligned}
$$

is very nearly confined between the values $f_{x}=2 / d$. [Note that $\operatorname{sinc} x=(\sin \pi x) / \pi x]$.

We sample this spectrum at intervals $1 / P d$, giving $4 P-1$ samples between the limits $\pm 2 / d$ (fig. 3 ), giving a sampled spectrum

$$
F_{\mathrm{s}}\left(f_{x}\right)=F\left(f_{x}\right) \text { comb } P d f_{x} \text {, }
$$

where comb $a f_{x}=\Sigma \delta\left(f_{x}-n / a\right)$ and $\delta$ is the Dirac delta function. The sampling results in a modified input function

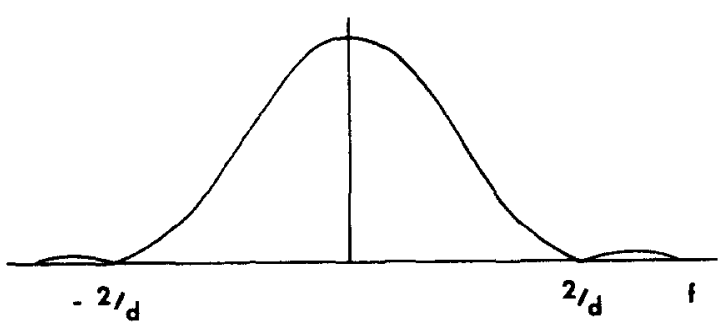

Fig. 3. Spatial frequency spectrum of pulse. Only the envelope is shown; 63 discrete frequencies reside under the envelope.

$$
\begin{aligned}
f_{\mathbf{s}}(x) & =\left[\left(\frac{1}{2}+\frac{1}{2} \cos 2 \pi x / d\right) \text { rect } x / d\right] * \operatorname{comb} x / P d \\
& =\sum_{-2 P}^{2 P} a_{n} \cos 2 \pi n x / d .
\end{aligned}
$$

This function, being composed of a finite number of harmonically-related spatial frequencies, thus physically corresponds to a set of $4 P-1$ plane waves, with incidence angles $\theta=\sin ^{-1} n \lambda / d$. These we propagate through the grating structure, using the Alferness method. Some of these components satisfy nearly or exactly the Bragg condition and produce diffracted waves at the output; others do not. At the output plane, or exiting surface, all incident components, along with the new components generated by diffraction, are combined, thus forming the output waveform.

Some precautions should be observed in designing the experiment. The sampling process forms a periodic function $f_{\mathrm{s}}$ from the single pulse $f$; thus, the input is a sequence of pulses of the form $f$, separated by intervals $P d$. We should choose $P$ sufficiently large, i.e., take sufficient samples, that, over the propagation distance, the pulse spreading due to diffraction does not result in an overlap of adjacent pulses. The greater the number of samples, $4 P-1$, the greater the separation distance between pulses.

The calculated results are shown in fig. 4. We have assumed a grating with a sinusoidal variation of refractive index, with index variation along the $x$ direction only (the unslanted fringe case). The pertinent parameters are: grating spacing $d=1 / 100 \mathrm{~mm}$, grating thickness $=0.54 \mathrm{~mm}$, wavelength $\lambda=6328 \AA$, and a refractive index of 1.50 . The program broke this into 18 thin gratings of thickness $0.03 \mathrm{~mm}$; such gratings satisfy Kogelnik's $Q$-factor criterion for a thin grating [3]. The output pulse is shown in a to $d$ for various 


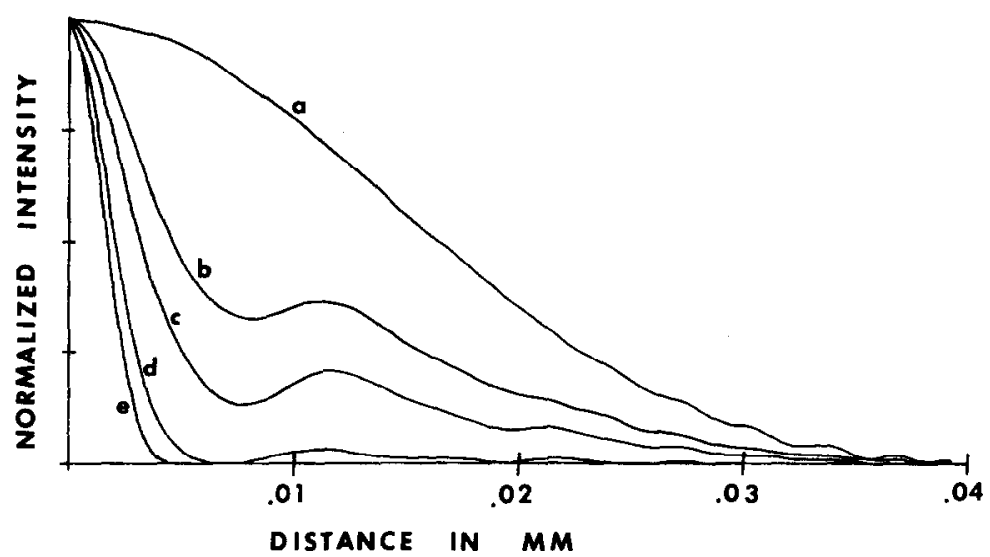

Fig. 4. Intensity distribution at output surface, for various values of $\Delta n$ : (a) $\Delta n=0$ (no grating); (b) $\Delta n=10^{-4} ;$ (c) $\Delta n=2 \times 10^{-4}$; (d) $\Delta n=6 \times 10^{-4}$; (e) pulse incident at input surface.

index variations. Curve e shows, for comparison, the incident pulse. Curve a shows the output pulse for $\Delta n=0$; this is just the diffraction spreading that occurs in the absence of a grating. Curve $b, c$, and $d$ show the output for increasing values of $\Delta n ;$ as $\Delta n$ increases, the pulse becomes increasingly confined. However, the greatest confinement appears to be of the energy that, in the absence of the grating structure, falls in the middle regions of the spread-out pulse. The energy in the outer portions, corresponding to the most oblique plane wave components, shows a. lesser tendency to confinement.

The plane waves satisfying the Bragg condition are represented by the spatial frequency components $\pm 1 / 2 d$, which is only $\frac{1}{4}$ of the maximum spatial frequency $2 / d$. Also, we note that the value of $\Delta n$ reported by Rosenberg and Chandross in their experiments was $6.4 \times 10^{-4}$; this is close to the value of $\Delta n\left(6 \times 10^{-4}\right)$ for which our curves show nearly complete wave confinement. Finally, we observe that thinner structures, such as dichromated gelatin, produce refractive index changes up to two orders of magnitude greater than those considered here. Thus, when spatial frequencies on the order of a few hundred cycles/mm are recorded, the guiding conditions are generally satisfied, even though the guiding effects are not observable, since the propagation distances are too short to reveal this phenomenon.

We thank the Office of Naval Research for their support of this work (contract number N 00014-67A-0181-0058).

\section{References}

[1] R.L. Rosenberg and E.A. Chandross, Appl. Opt. 10 (1971) 1986.

[2] R.L. Rosenberg, J. Opt. Soc. Am. 62 (1972) 1175.

[3] H. Kogelnik, Bell. Syst. Techn. J. 48 (1969) 2909.

14] R.C. Alferness, Appl. Phys., in press. 